\title{
Management of the Prosthetic Valve Thrombosis: This Time Is Different
}

\author{
Zineb Kounka1, Benjamín Roque-Rodríguez¹, Luis Enrique Lezcano-Gort'1, \\ David Antonio Chipayo-Gonzáles' ${ }^{1}$ Elena Gallego-Curto², Cecilio Raúl Rodríguez-Carreras ${ }^{1}$
}

\author{
${ }^{1}$ Department of Cardiology, Complejo Hospitalario Universitario de Cáceres, Cáceres, Spain \\ ${ }^{2}$ Intensive Care Unit, Complejo Hospitalario Universitario de Cáceres, Cáceres, Spain \\ Email: zineb.kounka@salud-juntaex.es, zanoubacadiz@hotmail.com
}

How to cite this paper: Kounka, Z., Roque-Rodríguez, B., Lezcano-Gort, L.E., Chipayo-Gonzáles, D.A., Gallego-Curto, E. and Rodríguez-Carreras, C.R. (2021) Management of the Prosthetic Valve Thrombosis: This Time Is Different. Case Reports in Clinical Medicine, 10, 185-190.

https://doi.org/10.4236/crcm.2021.107023

Received: June 2, 2021

Accepted: July 6, 2021

Published: July 9, 2021

Copyright (c) 2021 by author(s) and Scientific Research Publishing Inc. This work is licensed under the Creative Commons Attribution International License (CC BY 4.0).

http://creativecommons.org/licenses/by/4.0/

\begin{abstract}
Prosthetic valve thrombosis (PVT) is a rare but serious entity. Its diagnosis and treatment can be challenging and mortality remains high regardless of its management. A 50-year-old man admitted with cardiac arrest, whose physical examination and echocardioscopy suggested PVT confirmed with fluoroscopy. Intra-aortic fibrinolysis was the treatment chosen with successful results.
\end{abstract}

\section{Keywords}

Prosthetic Valve Thrombosis, Intra-Aortic Fibrinolysis, Low-Dose Slow Infusion

\section{Introduction}

Prosthetic valve thrombosis (PVT) is a rare and life-threatening complication with high mortality. In suspected PVT, a careful physical examination may reveal a muffling or disappearance of prosthetic sounds and the appearance of a new regurgitant or obstructive murmur [1]. Rapid confirmation of the diagnosis and prompt treatment is needed [1]. Delayed diagnosis and treatment can lead to increase morbidity and mortality. The therapeutic strategy (surgery or fibrinolysis) is mainly dependent on the hemodynamic status of the patient [2].

\section{Case Report}

A 50-year-old man received an aortic valve replacement five years earlier with a mechanical valve prosthesis with Dacron tube, due to a bicuspid aortic valve and type A aortic dissection detected incidentally by echocardiography (Figure 1). 
Since then, he is receiving Vitamin $\mathrm{K}$ antagonist (VKA). He also has permanent $\mathrm{AF}$ and an episode of acute pericarditis eight years ago.

He was admitted with out-of-hospital cardiac arrest (OHCA) and ventricular fibrillation as initial rhythm. Recovered spontaneous circulation after 2 minutes of advanced cardiopulmonary resuscitation. Physical examination highlighted the absence of clicking prosthetic valve sounds. Postresuscitation ECG showed atrial fibrillation (AF) with average ventricular rate around $140 \mathrm{bpm}$ with right bundle branch block (RBBB) and blood pressure of $90 / 60 \mathrm{mmHg}$ on norepinephrine infusion at $1.07 \mathrm{mcg} / \mathrm{Kg} / \mathrm{min}$. Analytically highlighted a metabolic acidosis with $\mathrm{pH} 7.08$, lactic acid $4.4 \mathrm{mmol} / \mathrm{L}$, bicarbonate $19.6 \mathrm{mmol} / \mathrm{L}$, base excess $-11.5 \mathrm{mmol} / \mathrm{L}$. The INR was subtherapeutic (1.4).

OHCA may be the initial manifestation of cardiac disease, particularly coronary artery disease. The physical examination and the patient's medical history make this diagnosis unlikely and make us suspect acute prosthetic valve thrombosis (PVT).

In the echocardioscopy, the absence of the prosthetic discs motion was striking. It also showed a suggestive image of thrombus in the left ventricular outflow tract (Figure 2). The left ventricular ejection fraction was mildly depressed (visual estimation 50\%) and right ventricular size and function are normal.
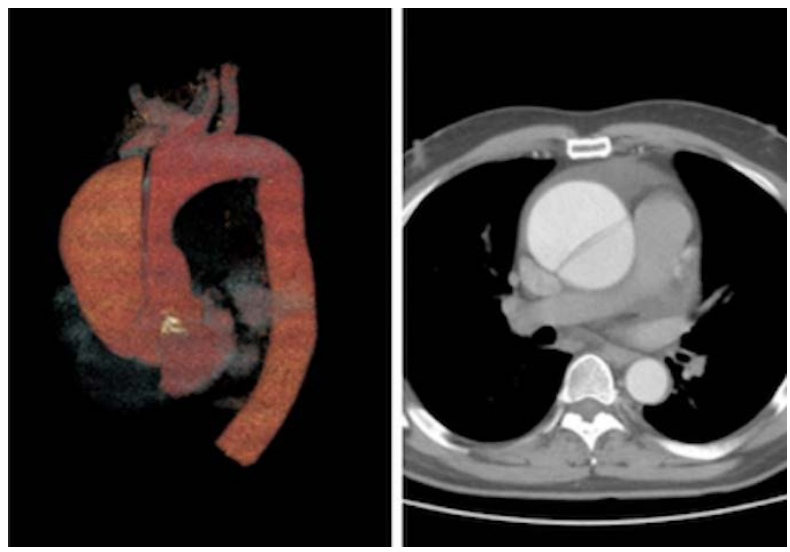

Figure 1. CT angiography. Type A aortic dissection.

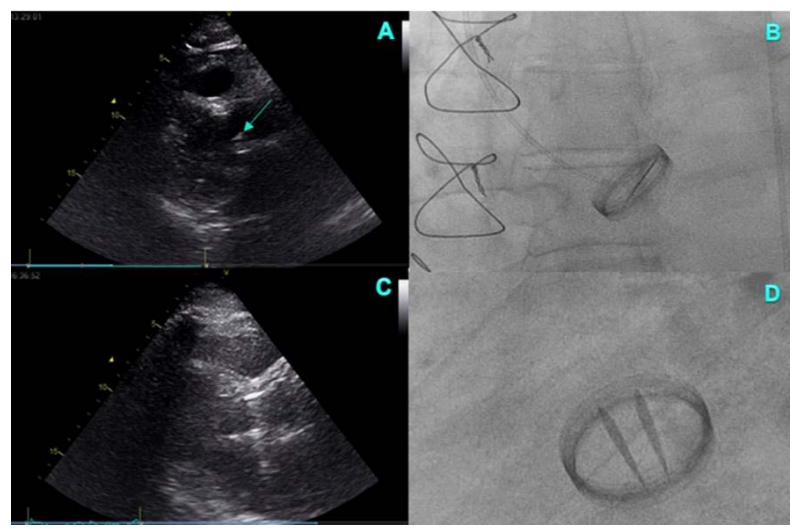

Figure 2. (A) and (B) pre-fibrinolysis. Arrow: Suggestive image of thrombus in the LVOT. (C) and (D) post-fibrinolysis. 
The patient suffered rapid hemodynamic deterioration (BP 43/29 $\mathrm{mmHg}$ ) and signs of peripheral hypoperfusion despite increasing doses of norepinephrine $(2.0 \mathrm{mcg} / \mathrm{kg} / \mathrm{min})$.

Emerging cinefluoroscopy (CFS) was performed showing the absence of motion of one of the prosthetic leaflets. Coronary angiography showed epicardial coronary arteries without obstructive lesions and slow flow.

Due to the critical situation, intra-aortic fibrinolysis with bolus of $10 \mathrm{mg}$ of rt-PA directed with the same diagnostic catheter was performed. Subsequently, the prosthetic valve was reevaluated, showing the correct motion of both leaflets, evidencing immediate hemodynamic improvement (Figure 3). ECG post-fibrinolysis showed disappearance of the RBBB (Figure 4). Slow infusion of $15 \mathrm{mg}$ of rt-PA was continued for 6 hours in addition to unfractionated heparin with target aPTT of 50 - 60 seconds. At 20 hours after admission, due to several episodes of asystole and hypotension, as well as echocardiographic data of prosthetic valve restenosis, fibrinolysis was repeated with rt-PA (slow infusion of $25 \mathrm{mg}$ over 6 hours), with favorable results within a few hours. The patient evolved favorably. Serial transthoracic echocardiography (TTE) showed a normally functioning prosthetic valve. Transesophageal echocardiography (TEE) showed severe spontaneous echo contrast in the left atrium with no thrombus seen.

CT angiography was performed and ruled out related abnormalities in the Dacron aortic tube. Cardiac MRI showed no thrombus or valvular pathology, unveiling changes secondary to previous aortic surgery and septal perfusion defects.

No systemic complications secondary to fibrinolysis were observed. The patient presented complete recovery of anoxo-ischemic encephalopathy, without neurological sequelae. At discharge he was on VKA.

At the 6-month follow up, our patient remained asymptomatic and optimally anticoagulated with VKA (time in therapeutic range 100\%).

\section{Discussion}

The annual rate of prosthetic valve thrombosis with mechanical valves ranges from $0.1 \%$ to $5.7 \%$ [1], a figure that rises up to $6 \%$ in patients with infra-therapeutic anticoagulation ranges [1], as in our case presenting the patient with INR 1.4. Tricuspid PVT is 20 times more frequent than the left-sided prostheses, and mitral PVT is 2 to 3 times more frequent than aortic position [2]. Mortality associated with PVT is high, between $10 \%$ and 33\% in the different studies.

The pathophysiology of PVT attends to the Virchow triad [2] and although PVT can manifest acutely, it is most often a subacute or chronic phenomenon [2]. The clinical presentation is variable and depends on the presence or absence of obstruction. A severe obstruction is associated with overt heart failure; however, a non-obstructive PVT is an accidental finding or may manifest as an embolic episode [2] [3]. 


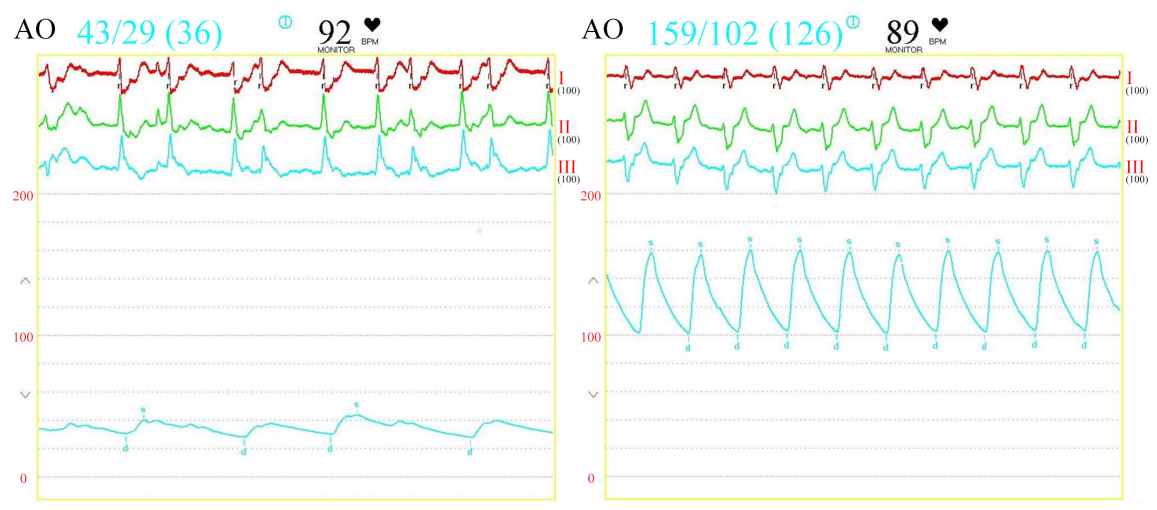

Figure 3. Left: Pre-fibrinolysis intra-aortic pressure curve. Right: Intra-aortic pressure curve immediately after intra-aortic fibrinolysis.

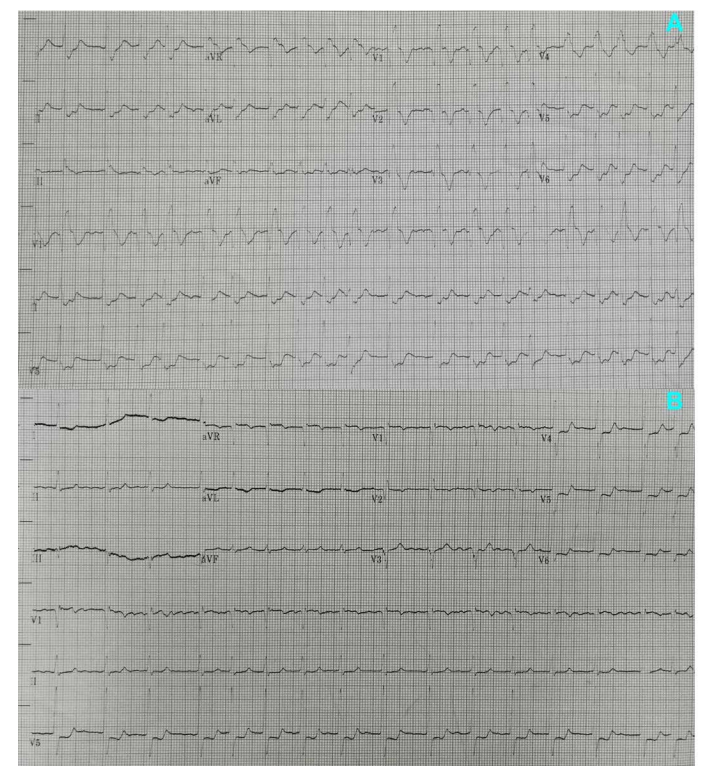

Figure 4. ECG. (A) After fibrinolysis. Atrial fibrillation and complete RBBB. (B) After Intra-aortic fibrinolysis. AF and disappearance of the RBBB.

In the case of our patient, he began with cardiorespiratory arrest, rare form of PVT presentation.

When PVT is suspected, a thorough physical examination should be performed with special attention to prosthetic sounds (decrease or disappearance) and the appearance of new murmurs (regurgitation or obstruction).

The urgent evaluation is indicated with TTE, TEE, fluoroscopy, and/or multidetector CT imaging to assess valve function, leaflets motion, and the presence of thrombus (Class of Recommendation (COR) 1, Level of Evidence (LOE) B) [3]. As for us, the first diagnostic test performed was a TTE, later confirmed by CFS. TEE can be performed when suspicion for PVT remains high after inconclusive TTE, but in hemodynamically unstable patients it is rarely necessary. The TEE was performed after stabilization of the patient to rule out intracardiac thrombogenic foci, visualizing severe spontaneous echo contrast in the left 
atrium, which made us suspect that the origin of the thrombus leading to valve obstruction could have been the left atrium and especially the left atrial appendage, due to the acute presentation.

The differential diagnosis is with pannus or fibrous tissue [2] [3]. In our case, acute presentation with hemodynamic instability and immediate improvement after fibrinolysis, made it unlikely.

Obstructive PVT requires urgent and aggressive treatment. Although the treatment recommendations in the literature are limited, the two available therapeutic possibilities are surgery and fibrinolysis. According to 2020 ACC/AHA Guideline for the Management of Patients With Valvular Heart Disease, urgent initial treatment with either slow-infusion, low-dose fibrinolytic therapy or emergency surgery is recommended (COR 1, LOE B) [3]. European point of view 4 is different, where surgery is recommended for obstructive thrombosis in critically ill patients without serious comorbidity (COR I, LOE C) and fibrinolysis (using rt-PA $10 \mathrm{mg}$ bolus $+90 \mathrm{mg}$ in $90 \mathrm{~min}$ with UFH or streptokinase $1,500,000 \mathrm{U}$ in $60 \mathrm{~min}$ without UFH) should be considered when surgery is not available or is very high risk or for thrombosis of right-sided prostheses (COR IIa, LOE C) [4].

The decision between surgery and systemic fibrinolysis for symptomatic left-sided PVT should be individualized, depending on the type of prosthesis and its location, but mainly on the hemodynamic status of the patient.

Surgery is also reasonable in the presence of large or mobile thrombi and fibrinolysis is reserved for patients with a worse functional class and for those in centers that do not have cardiac surgery [1].

As far as we are concerned, fibrinolysis was the chosen treatment since the patient debuted with cardiac arrest, and was in NYHA functional class IV in a hospital without on-site cardiac surgery.

The fibrinolysis strategy we used was the slow-infusion (6 hours) with low-dose (25 mg) which we have modified, adding initial intra-aortic bolus (10 mg), given the critical initial situation of the patient, and the remaining dose $(15 \mathrm{mg})$ in slow-infusion. In the TROIA trial [5], the regimen of low-dose slow infusion of $\mathrm{r}$-tPA repeated as needed provides effective and safe thrombolysis in patients with prosthetic valve thrombosis. Recent studies using an echocardiogram-guided, slow-infusion, low-dose fibrinolytic protocol have shown hemodynamic success rates $>90 \%$, with embolic event rates $<2 \%$ and major bleeding rates $<2 \%[6]$.

\section{Conclusion}

Mortality associated with PVT is high, regardless of the treatment used. The success of the treatment will depend firstly on the high clinical suspicion based on the physical examination and the hemodynamic status, and secondly on the rapid confirmation of the diagnosis through CFS or echocardiography. The therapeutic strategy is conditioned by the type of prosthesis and its location, but mainly by the hemodynamic status of the patient. 


\section{Conflicts of Interest}

The authors declare no conflicts of interest regarding the publication of this paper.

\section{References}

[1] Nishimura, R.A., Otto, C.M., Bonow, R.O., et al. (2017) AHA/ACC Focused Update of the 2014 AHA/ACC Guideline for the Management of Patients with Valvular Heart Disease: A Report of the American College of Cardiology/American Heart Association Task Force on Clinical Practice Guidelines. Circulation, 135, e1159-e1195.

[2] Roudaut, R., Serri, K. and Lafitte, S. (2007) Thrombosis of Prosthetic Heart Valves: Diagnosis and Therapeutic Considerations. Heart, 93, 137-142.

https://doi.org/10.1136/hrt.2005.071183

[3] Otto, C.M., Nishimura, R.A., Bonow, R.O., et al. (2020) ACC/AHA Guideline for the Management of Patients with Valvular Heart Disease: A Report of the American College of Cardiology/American Heart Association Joint Committee on Clinical Practice Guidelines. Circulation, 143, e35-e71.

https://doi.org/10.1161/CIR.0000000000000932

[4] Baumgartner, H., Falk, V., Bax, J.J., et al. (2017) ESC/EACTS Guidelines for the management of Valvular Heart Disease. European Heart Journal, 38, 2739-2791.

[5] Özkan, M., Gündüz, S., Biteker, M., et al. (2013) Comparison of Different TEE-Guided Thrombolytic Regimens for Prosthetic Valve Thrombosis: The TROIA Trial. JACC Cardiovasc Imaging, 6, 206-216. https://doi.org/10.1016/j.jcmg.2012.10.016

[6] Özkan, M., Gündüz, S., Gürsoy, O.M., et al. (2015) Ultraslow Thrombolytic Therapy: A Novel Strategy in the Management of PROsthetic MEchanical Valve Thrombosis and the prEdictors of outcomE: The Ultra-Slow PROMETEE Trial. American Heart Journal, 170, 409-418. https://doi.org/10.1016/j.ahj.2015.04.025 